\title{
Erstellung von Referenzbereichen für $\alpha$-Amylase und Lipase bei Neugeborenen, Säuglingen, Kindern und Jugendlichen*
}

\section{Reference values for $\alpha$-amylase and lipase in neonates, children and infants}

Gudrun Wiedemann, D. Wetzel

Klinisch-Chemisches Labor der Klinik und Poliklinik für Kindermedizin, des Klinikums Erfurt

\section{Zusammenfassung:}

Bei 916 bzw. 742 gesunden Neugeborenen, Säuglingen, Kindern und Jugendlichen im Alter von 5 Tagen bis 18 Jahren wurde die Aktivität der $\alpha$-Amylase und Lipase im Serum bestimmt. Das Probandenkollektiv wurde in neun Referenzgruppen aufgeteilt. Nach den Empfehlungen der International Federation of Clinical Chemistry wurde der 95\%Streubereich als Referenzbereich angegeben. Normalverteilungen nach Gauss ließen sich nur in sehr wenigen Referenzgruppen nachweisen, deshalb wurde in allen Referenzgruppen neben der 50. Perzentile die 2,5. und 97,5. Perzentile berechnet. Nach Prüfung auf signifikante Unterschiede zwischen den einzelnen Referenzgruppen mittels U-Test nach Mann \& Whitney und erneuter Zusammenfassung der Gruppen wurden Referenzbereiche für die Aktivität der $\alpha$-Amylase und Lipase im Serum ermittelt

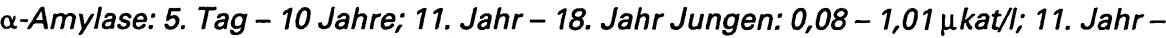
18. Jahr Mädchen: , 0,12-0,99 $\mu \mathrm{kat} / \mathrm{l}$.

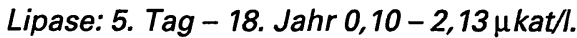

\section{Schlüsselwörter:}

Referenzbereiche - Neugeborene - Säuglinge - Kinder - Jugendliche - Amylase Lipase

\section{Summary:}

In a total of 916 neonates, infants and children, the activity of $\alpha$-amylase in serum was determined. In a total of 742 neonates, infants and children, the activity of lipase in serum was determined. Nine reference groups were established. According to the recommendationes of the international Federation of Clinical Chemistry the reference ranges were defined as 95\%-range between the 2.5th and the 97.5th percentile. Differences between reference groups were determined using the $U$-test by Mann \& Whitney. The groups were summarized if possible. The following reference ranges were found

$\alpha$-amylase: 5 th day - 10th day; 11th year - 18th year boys: $0.08-1.01 \mu \mathrm{kat} / \mathrm{l} ; 11$ th year - 18th year girls: $0.12-0.99 \mu \mathrm{kat} / \mathrm{l}$.

lipase: 5 th day - 18 th year $0.10-2.13 \mu \mathrm{kat} / \mathrm{l}$.

Keywords:

Reference ranges - neonates - children - infants - $\alpha$-amylase - lipase

\footnotetext{
* Herrn Prof. Dr. H. Baufeld zum 75. Geburtstag gewidmet
} 


\section{Sy్s!mex SE-9000: Der Imnovmitionsschub bei aufomm tischen Hämafiologiesystemen:}
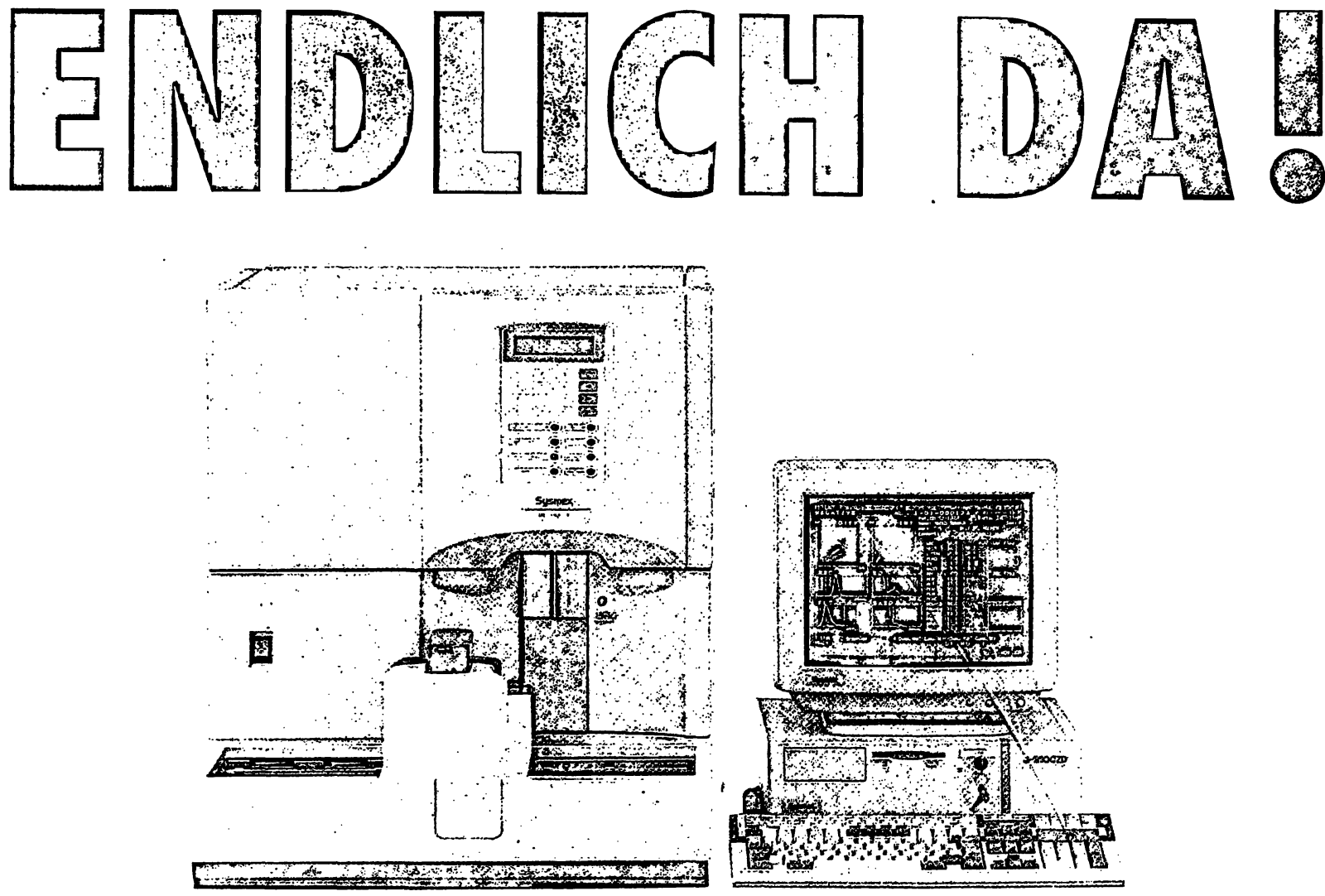

Sysmex SE-9000: Innovationsschub für mehr Wirtschaftlichkeit und Sicherheit.

Innovationen bei Hämatologiesystemen sind für den Anwender nur dann nutzenrelevant, wenn sie im Endergebnis die Wirtschaftlichkeit und Analysensicherheit nachhaltig steigern.

Der Sysmex SE-9000 ist gegenüber vergleichbaren Systemen nicht nur erheblich kompakter, sondern in den wesentlichen Features auch signifikant leistungsfähiger. Nachfolgend nur 3 Beispiele für Leistungsüberlegenheit durch Innovation:

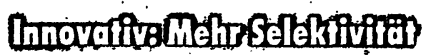

Die beliebige Selektivität der Analyse nach Großem und Kleinem Blutbild (durch Barcode, Arbeitsliste oder On-line Request-Verfahren) spart Reagenzien und erübrigt das Vorsortieren der Proben.

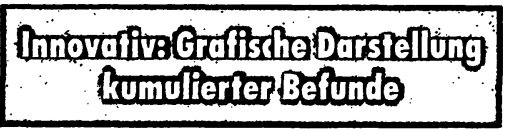

Kumulierte Patientenbefunde werden nicht - mehr nur numerisch, sondern auch grafisch mit Scattergramm und Histogramm dargestellt. Damit werden Entwicklungen und Prozesse auf einen Blick erfaßbar.

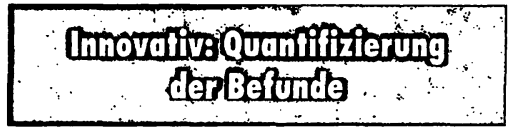

Wurden bisher pathologische Befunde nur qualitativ signalisiert, so mißt jetzt ein separater Kanal auch die Quantität der befallenen Zellen. Das Ausmaß einer pathologischen Entwicklung wird damit optimal erkennbar.

\section{Ich will mehr wissen!}

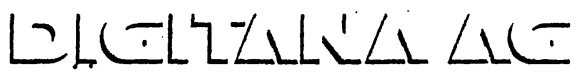

Ganz genau und zuverlïssig.

D - 22083 Hamburg - WeidestriaBe 118 b - Tel.: $040 / 2707050$ $\mathrm{CH}-8810$ Horgen - Burghaldenstraße 11- Tel.: $01 / 7256191$ 


\section{Einführüng}

Die Bestimmung der, $\alpha$-Amylase und Lipase im Serum ist ein anerkanntes diagnostisches Hilfsmittel bei Pankreaserkrankungen. Bei Erkrankungen der Speicheldrüsen ist die Bestimmung der $\alpha$-Amylase ebenfalls ein wichtiger diagnostischer Parameter.

\section{Ziel der Untersuchung}

\section{Das Ziel der Untersuchung bestand in}

- der Ermittlung von Referenzbereichen für die Aktivität der Serumenzyme $\alpha$-Amylase und Lipase bei gesunden Neugeborenen, Säuglingen, Kindern und Jugendlichen

- der Prüfung auf signifikante Unterschiede zwischen den Referenzgruppen.

\section{Material und Methode}

Von 916 bzw. 742 gesunden Neugeborenen, Säuglingen, Kindern und Jugendlichen im Alter zwischen 5 Tagen und 18 Jahren wurde die Enzymaktivität der $\alpha$-Amylase und Lipase im Serum bestimmt. Bei Neugeborenen wurde im Rahmen des Hypothyreose-Screenings Venenblut entnommen. Für die Blutentnahme bei allen anderen Probanden lag das schriftliche Einverständnis der über den Zweck der Untersuchung aufgeklärten Eltern vor. Die Ethikkommission der Medizinischen Hochschule Erfurt hatte dieser Vorgehensweise zugestimmt.

Tab. 1: Altersstruktur des Probandenkollektivs

\begin{tabular}{|l|l|c|c|}
\hline Gruppe & Alter - & $\alpha$-Amylase & Lipase \\
\hline & & $n$ & $n$ \\
\hline 1 & $5 . \quad$ Tag & 133 & 104 \\
\hline 2 & $6 .-28 . \mathrm{Tag}$ & 49 & 42 \\
\hline 3 & $2 .-6 . \mathrm{Monat}$ & 111 & 88 \\
\hline 4 & 7.-12.Monat & 46 & 36 \\
\hline 5 & $2 .-4 . \mathrm{Jahr}$ & 193 & 141 \\
\hline 6 & 5.- 7.Jahr & 87 & 85 \\
\hline 7 & $8 .-10 . \mathrm{Jahr}$ & 93 & 86 \\
\hline 8 & $11 .-18 . \mathrm{Jahr}, 0^{\circ}$ & 113 & 86 \\
\hline 9 & $11 .-18 . \mathrm{Jahr}, Q$ & 91 & 74 \\
\hline
\end{tabular}

Eine Übersicht zur Alterszusammensetzung des Probandenkollektivs gibt Tabelle 1. Bei der Entscheidung über die Aufnahme oder den Ausschluß von Probanden galten die von Witt und Trendelenburg (1) und der IFCC (7) erarbeiteten Ausschlußkriterien. In die Gruppe am 5. Lebenstag wurden nur Neugeborene mit einem Geburtsgewicht zwischen 2500 und $4000 \mathrm{~g}$ und einem Gestationsalter zwischen vollendeter 37 . und vollendeter 41. Woche aufgenommen. Neugeborene mit einer Hyperbilirubinämie wurden ausgeschlossen. Bei den Müttern der Neugeborenen durfte keine akute oder chronische Erkrankung festgestellt worden sein.

\section{Probenmaterial}

Etwa 2 ml Blut wurde durch Punktion einer Schädel- oder Armvene unterVerwendung von Sicherheitsmonovetten der Firma Sarstedt, Nümbrecht abgenommen. Unmittelbar danach erfolgte die Zentrifugation bei $3000 \mathrm{U} / \mathrm{min}$ für die Dauer von 5 Minuten. Das Serum wurde abpipettiert und bei $-22^{\circ} \mathrm{C}$ bis zur Bestimmung der einzelnen Parameter eingefroren.

\section{Methoden}

Die Bestimmung der $\alpha$-Amylase im Serum erfolgte unter Véwendung des Synchron CX Amylase-Reagenzien Kits (Maltotetraose) der Firma Beckman und die der Lipase mit Hilfe des Monotests "Lipase" (Triolein) von Boehringer Mannheim. Alle Untersuchungen wurden am mechanisierten Analysensystem Synchron- CX 7 der Firma Beckman bei $37^{\circ} \mathrm{C}$ durchgeführt.

Zur Kontrolle der Präzision von Tag zu Tag wurden für die Enzyme in jeder Serie die Kontrollseren Serodos und Serodos Plus der Firma Greiner, Flacht mitgeführt. Aus den jeweiligen Einzelergebnissen wurden für jedes Enzym der arithmetische Mittelwert $(\bar{x})$, die Standardabweichung (s) und der Variationskoeffizient (VK) als Maß für den relativen methodischen Fehler errechnet.

\section{Statistische Auswertung der Ergebnisse}

Zunächst wurden die Ergebnisse in den einzelnen Altersgruppen in Histogrammen dargestellt. Eine Prüfung des Verteilungstyps wurde mit Hilfe der Kolmogorov-Smirnov-Tests vorgenommen. Unterschritt die so ermittelte Irrtumswahrscheinlichkeit den vorgegebenen Wert von $\alpha=0,05$, so wurde eine Normalverteilung nach Gauss angenommen. Bei Nichtvorliegen einer Gauss'schen Normalverteilung wurde die 2,5.,50. und 97,5. Perzentile in den Referenzgruppen ermittelt. Mit Hilfe des U-Tests nach Mann \& Whitney wurden die Altersgruppen für jedes Enzym auf signifikante Gruppenunterschiede untersucht. Bestand kein signifikanter Unterschied zwischen den Referenzgruppen, wurde in folgendem diese als eine gemeinsame Gruppe 


\section{Originalie}

Tab. 3: Referenzbereiche für die Aktivität der Lipase bei 37 C im Serum bei Neugeborenen, Säuglingen, Kindern und Jugendlichen (An-gaben in $\mu$ kat/I)

\begin{tabular}{|l|l|l|l|l|l|}
\hline Altersgruppe & $\mathrm{n}$ & Median & Referenzbereich & Minimum & Maximum \\
\hline & & $(50 . \mathrm{PZ})$ & $\left(2.5-97.5 \mathrm{PZ}^{*}\right)$ & & \\
\hline (1) 5. Tag & 104 & 0.78 & $0.11-2.29 * * *$ & 0.05 & 2.73 \\
\hline (2) 6.- 28.Tag & 42 & 0.80 & $0.03-2.69 * * *$ & 0.02 & 2.73 \\
\hline (3) 2.- 6.Monat & 88 & 0.75 & $0.05-2.32^{* * *}$ & 0.01 & 2.48 \\
\hline (4) 7.- 12.Monat & 36 & 0.90 & $0.30-2.08 * *$ & 0.23 & 2.61 \\
\hline (5) 2.- 4.Jahr & 141 & 0.76 & $0.10-1.88$ & 0.05 & 1.97 \\
\hline (6) 5.- 7.Jahr & 85 & 0.83 & $0.06-2.09$ & 0.02 & 2.31 \\
\hline (7) 8.- 10.Jahr & 86 & 0.79 & $0.05-2.64$ & 0.03 & 3.76 \\
\hline (8) 11.- 18.Jahr, $\sigma^{*}$ & 86 & 0.78 & $0.06-2.08$ & 0.02 & 2.14 \\
\hline (9)11.- 18.Jahr, 9 & 74 & 0.85 & $0.07-2.10$ & 0.13 & 2.13 \\
\hline
\end{tabular}

Tab. 4: Ergebnisse der Kontrolle der Präzision von Tag zu Tag für dieEnzyme $\alpha$-Amylase und Lipase im Serum bei $37^{\circ} \mathrm{C}$

\begin{tabular}{|l|l|l|l|l|l|}
\hline Kontrollserum & Parameter & $\mathrm{n}$ & $\overline{\mathrm{x}}$ & $\mathrm{s}$ & $\mathrm{VK}$ \\
\hline & $\mu \mathrm{kat} / 1$ & & $\mu \mathrm{kat} / 1$ & $\mu \mathrm{kat} / 1$ & $(\%)$ \\
\hline \multirow{2}{*}{ Serodos } & $\alpha$-Amylase & 30 & 0.90 & 0.02 & 1.67 \\
\cline { 2 - 6 } & Lipase & 28 & 0.92 & 0.04 & 4.69 \\
\hline $\begin{array}{l}\text { Serodos } \\
\text { Plus }\end{array}$ & $\alpha$-Amylase & 30 & 1.66 & 0.02 & 1.45 \\
& & & & & \\
\cline { 2 - 6 } & Lipase & 30 & 4.62 & 0.11 & 2.44 \\
\hline
\end{tabular}

Tab. 5: Referenzbereiche für die Aktivität der $\alpha$-Amylase im Serum aus der Literatur

\begin{tabular}{|c|c|c|c|c|c|}
\hline Autor & Methode & $\begin{array}{l}\text { Angabeen zu den } \\
\text { Altersgruppen }\end{array}$ & $\begin{array}{l}\text { Angaben ax } \\
\text { Arrahl der } \\
\text { Probeaden }\end{array}$ & $\begin{array}{l}\text { Angaben zu } \\
\text { Verteilungatyp } \\
\text { und : } \\
\text { Strewbereich }\end{array}$ & Referenbereich \\
\hline $\begin{array}{l}\text { Struckmeyer } \\
\text { und Haid } \\
\text { (4) }\end{array}$ & $\begin{array}{l}\text { Farbiest mit } \\
\text { Froidecaing } \\
\text { von p-Nituophenol }\end{array}$ & 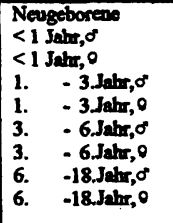 & $\begin{array}{l}\text { Keine } \\
\text { Angabe }\end{array}$ & $\begin{array}{l}\text { Median } \\
\text { 95\% Streubereich }\end{array}$ & $\begin{array}{l}10,5(2,1-29,8) \cup / \\
11,6(4,6-28,1) \cup n \\
12,8(6,9-27,4) \cup / \\
40,2(16,5-61,0) \cup / \\
34,6(13,5-50,1) \cup n \\
37,7(7,7-53,5) \cup / \\
34,4(11,6-50,8) \cup n \\
34,4(18,8-50,8) \cup n \\
32,9(9,8-50,8) \cup n\end{array}$ \\
\hline $\begin{array}{l}\text { Zabranaky und } \\
\text { Tritschler } \\
\text { (5) }\end{array}$ & $\begin{array}{l}\text { p-Nitrophenyl-a, } \\
\text { D-maliohepteosid } \\
37{ }^{\circ} \mathrm{C}\end{array}$ & $\begin{array}{ll}3 & -353 \text { Tage } \\
1 & -16 \text { Jahre }\end{array}$ & $\begin{array}{r}30 \\
186\end{array}$ & $\begin{array}{l}\text { 80\%berw.95\% } \\
\text { Streabereich }\end{array}$ & $\begin{array}{r}-60 \mathrm{U} / 1 \\
40-200 \mathrm{U}\end{array}$ \\
\hline $\begin{array}{l}\text { Gillend, Simbela } \\
\text { und Ooodglick } \\
\text { (2) }\end{array}$ & $\begin{array}{l}\text { modinizierte Nolaca- } \\
\text { Somogvi-Methode }\end{array}$ & $\begin{aligned} & 3-2 \text { Monite } \\
& 7-6 \text { Monite } \\
& 9-8 \text { Monate } \\
& 13-12 \text { Monate } \\
& 19-18 \text { Monite } \\
& 19-35 \text { Monate } \\
& 3-4 \text { Jalure } \\
& 5-12 \text { Jehre } \\
& 13-19 \text { J live } \\
&\end{aligned}$ & $\begin{array}{l}\mathbf{5 3} \\
\mathbf{2 0} \\
\mathbf{2 3} \\
\mathbf{4 1} \\
\mathbf{2 8} \\
\mathbf{7 0} \\
\mathbf{6 3} \\
\mathbf{9 1} \\
\mathbf{6 5} \\
\end{array}$ & $\begin{array}{l}\text { 90*-Steubereich } \\
\text { Modinn }\end{array}$ & $\begin{array}{r}2,3(0,5-6,3) \cup / \\
4,6(1,8-9,1) \cup / \\
5,6(2,3-12,6) \cup / \\
7,3(2,9-15,5) \cup / \\
7,3(3,5-17,9) \cup / \\
10,7(5,0-21,3) \cup / \\
13,7(7,2-24,3) \cup / 1 \\
15,1(8,5-26,6) \cup / \\
14,4(8,0-26,4) \cup /\end{array}$ \\
\hline $\begin{array}{l}\text { Inselepital Bem } \\
\text { (3) }\end{array}$ & keine Angabe & $\begin{array}{l}-1 \mathrm{Jahr} \\
-14 . \mathrm{Jabr}\end{array}$ & $\begin{array}{l}\text { keing } \\
\text { Angube }\end{array}$ & $\begin{array}{l}\text { keine } \\
\text { Angibe }\end{array}$ & $\begin{array}{l}-160 \mathrm{U} / \\
-160 \mathrm{U} /\end{array}$ \\
\hline $\begin{array}{l}\text { Arbeitsenuppe } \\
\text { Pldiatrische } \\
\text { Klimiache Chemie } \\
\text { (9) }\end{array}$ & $\begin{array}{l}\text { Fatied nit } \\
\text { Freisctangs } \\
\text { von p-Nitrophenol } \\
\text { (Behring) }\end{array}$ & $\begin{array}{l}\text { Seuginge } \\
\text { atere Kindor }\end{array}$ & $\begin{array}{l}\text { keine } \\
\text { Angabe }\end{array}$ & $\begin{array}{l}\text { keine } \\
\text { Angabe }\end{array}$ & $\begin{array}{l}-40 \mathrm{UI} \\
-49 \mathrm{UI} \\
.\end{array}$ \\
\hline
\end{tabular}


betrachtet, Medianwerte und Referenzbereiche neu berechnet.

\section{Ergebnisse}

Bei insgesamt 916 bzw. 714 gesunden Probanden wurde die Aktivität der $\alpha$-Amylase bzw. Lipase im Serum bestimmt. Eine Übersicht über die Ergebnisse in den einzelnen Gruppen vor der Signifikanzprüfung gibt Tabelle 2 und 3. Alle Referenzgruppen wurden auf signifikante Gruppenunterschiede mit Hilfe des U-Testes nach Mann \& Whitney geprüft. In allen zusammengefaßten Gruppen wurden die Medianwerte und Referenzbereiche für die Aktivität der $\alpha$-Amylase und Lipase im Serum neu berechnet.

Die Ergebnisse der Qualitätskontrolle zeigt Tabelle 4. Die intraseriellen Variationskoeffizienten lagen unter $5 \%$.

\section{Diskussion}

Frühere ermittelte Referenzbereiche finden sich im Schrifttum nur spärlich, Angaben dazu sind in den Tabellen 5 und 6 aufgeführt. Diese Angaben sind nicht oder nur bedingt mit den eigenen Ergebnissen vergleichbar:

- Die Alterseinteilung wurde anders getroffen.

- Unterschiedliche Meßtemperaturen kamen zur Anwendung.

- Angaben zur Probandenzahl sind teilweise nicht vorhanden.

- Es sind ungenügende Angaben zum Verteilungstyp gemacht.

- Die Wahl der Referenzbereiche ist unterschiedlich.

Zur Ermittlung von Referenzbereichen im Kindesalter wurden 916 bzw. 714 Neugeborene, Säuglinge, Kinder und Jugendliche vom 5 . Lebenstag bis zum vollendeten 18. Lebensjahr einbezogen. Die untersuchten Probanden wurden zunächst in jeweils 9 Gruppen nach dem Alter bzw. dem Geschlecht eingeteilt (Tabelle 1). Die gewählte Einteilung erfolgte in Anlehnung an die Vorschläge von Egger et al. (6) und die Empfehlungen der International Federation of Clinical Chemistry (7).

Da in der überwiegenden Zahl der Referenzgruppen für jedes Enzym keine Normalverteilungen der Referenzwerte vorlagen, erfolgte zur Ermittlung der Referenzbereiche neben der Angabe der Medianwerte die Berechnung der 2,5 . und 97,5 . Perzentile.

Wie bereits aus den Tabellen 2 und $3 \mathrm{zu}$ ersehen ist, unterscheiden sich die Referenzbereiche der beiden Enzyme $\alpha$-Amylase und Lipase im Serum vom 5 . Lebenstag bis zum vollendeten 18. Lebensjahr kaum. Die von der Arbeitsgruppe Pädiatrische Klinische Chemie (3) gewonnenen Ergebnisse für die Amylaseaktivität können bestätigt werden. Gillard, Simbala und Goodglick (2) fanden dagegen niedrigere Amylaseaktivitäten im frühen Kindesalter.

Für die Lipase ergab sich für das gesamte Kindesalter ein einheitlicher Referenzbereich (Tabelle 7), Temme et al. (8) dagegen fand niedrige Lipaseaktivitäten im Serum bei Neugeborenen und einen Aktivitätsanstieg mit zunehmendem Alter.

Bei der $\alpha$-Amylase war lediglich bei den über 10 jährigen Mädchen ein statistisch signigikanter Unterschied vorhanden, der aber für die Praxis zu vernachlässigen ist (Tabelle 7).

Tab. 7: Referenzbereiche für die Aktivität der $\alpha$-Amylase und Lipase im Serum von Kindern

\begin{tabular}{|c|c|}
\hline a-Amylase & \\
\hline $\begin{array}{l}\text { 5.Tag - 10.Jahr } \\
\text { 11.Jahr - 18.Jahr, } \sigma^{\circ}\end{array}$ & $0,08 \cdot 1,01$ p wan \\
\hline 11.Jahr - 18.Jahr, ? & $0,12-0,99$ wanl \\
\hline \multicolumn{2}{|l|}{ Upase } \\
\hline 5.Tag - 18.Jahr & $0,10-2,13$ нkan \\
\hline
\end{tabular}

Tab. 6: Referenzbereiche für die Aktivität der Lipase im Serum aus der Literatur

\begin{tabular}{|c|c|c|c|c|c|}
\hline Autor & Methode & $\begin{array}{l}\text { Angaben zu den } \\
\text { Alters- } \\
\text { guppen }\end{array}$ & $\begin{array}{l}\text { Angaben zur } \\
\text { Anzahl der } \\
\text { Probanden }\end{array}$ & $\begin{array}{l}\text { Angaben zu } \\
\text { Verteilungs- } \\
\text { typ und } \\
\text { Streubereich }\end{array}$ & Referenzbereich \\
\hline $\begin{array}{l}\text { Termme et al. } \\
\text { (8) }\end{array}$ & $\begin{array}{l}\text { Turbidimetrischer } \\
\text { Test mit } \\
\text { Triolein } \\
\text { "Alpha-Amylase- } \\
\text { PNP-BM" } \\
25^{\circ} \mathrm{C}\end{array}$ & $\begin{array}{l}\text { Tag } \\
2 \text { Tage } \\
3 \text { Tage - } 12 \text { Monate } \\
13 \text { Monate - } 16 \text { Jahr }\end{array}$ & $\begin{array}{r}21 \\
17 \\
13 \\
160\end{array}$ & $\begin{array}{l}\text { keine } \\
\text { Angabe }\end{array}$ & $\begin{aligned} & 0-15,5 \cup / 1 \\
& 4,9- 27,3 \cup / 1 \\
& 0,4- 67,7 \cup / 1 \\
& 2,0-78,3 \cup / 1\end{aligned}$ \\
\hline $\begin{array}{l}\text { Arbeitsgerppe } \\
\text { Padiatrische } \\
\text { Klimische Chemie } \\
\text { (9) }\end{array}$ & $\begin{array}{l}\text { Turbidimetrisch } \\
\text { (Bochringer) } \\
25^{\circ} \mathrm{C}\end{array}$ & $\begin{array}{l}\text { Neugeborene } \\
\text { altere Kinder }\end{array}$ & $\begin{array}{l}\text { keine } \\
\text { Angaben }\end{array}$ & $\begin{array}{l}\text { keine } \\
\text { Angabe } \\
\text {.. }\end{array}$ & $\begin{array}{l}-80 \mathrm{U} / \\
-\quad 115 \mathrm{U} /\end{array}$ \\
\hline
\end{tabular}




\section{Originalie}

Literatur:

1. Witt, J.; Trendelenburg, C. (1982): Gemeinsame Studie zur Erstellung von Richtwerten klinisch chemischer Kenngrößen im Kindesalter, J. Clin. Chem. Clin. Biochem. 20, 235 - 242

2. Gillard, B.K.; Simbala, J.A.; Goodglick, L. (1983): Reference intervals for amylase isoenzymes in serum and plasma of infants and children, Clin. Chem. $29,1119-1123$

3. Referenzwerte Chemisches Zentrallabor Inselspital, Bern (1984): $\alpha$-Amylase. In: Berner Datenbuch der Pädiatrie, aus der Medizinischen UniversitätsKinderklinik, Inselspital Bern; 4. Auflage, Stuttgart, Jena, New York, Gustav Fischer Verlag 1992, S. 718

4. Struckmeyer, H.; Haid, H. (1986): Richtwerte für das kinderärztliche Laboratorium; 1. Auflage, Marburg, Die Medizinische Verlagsgesellschaft

5. Zabransky, S.; Tritschler, W. (1986): $\alpha$-Amylase: Richtwerte bei Kindern, Klin. Pädiat. 198, $29-32$

6. Egger, E.; Dummler, W.; Neymeyer, H.G.; Schwarze, H.; Scholz, R. (1987): Vorschlag zum Arzneibuch der DDR, Diagnostische Laboratoriumsmethoden AB (D.L.) - DDR 89, Allgemeines, Referenzbereiche; Zent. bl. Pharm. 126, 737 $-747$

7. International Federation of Clinical Chemistry (1987): Scientific Committee Clinical Section Expert Panel on Theory of Reference Values and International Committee for Standardisation in Haematology. Standing Committee on Reference Values Approves recommendation (1987) on the theory of reference values. Part 5. Statistical treatment of collected reference values. Determination . of reference limits. J. Clin. Chem. Clin. Biochem. 25, $645-656$

8. Temme, H.; Führer, J.; Roka, L.; Otten, A.; Ringel, M.; Bödeker, R. (1989):

Referenzwerte für Lipase im Serum von Kindern und Methodenvergleich für
Amylase und Lipase im niedrigen Aktivitätsbereich. J. Clin. Chem. Clin. Biochem. 27, 9

9. Arbeitsgruppe Pädiatrische Klinische Chemie (1990): Amylase, Lipase. In: Pädiatrie in Praxis und Klinik, Band IV, 2. Auflage. Hrsg.; K.-D. Bachmann, H. Ewerbeck, E. Kleihauer, E. Rossi, G. Stalder, Stuttgart, New York. Gustav Fischer Verlag 1990 und Georg Thieme Verlag 1990, S. 336 - 380

\section{Danksagung:}

Wir danken Frau R. Biesenbach für die technische Assistenz. Außerdem sei der Firma Beckman für die großzügige Unterstützung gedankt, ohne die diese Arbeit nicht möglich gewesen wäre.

\section{Anschrift der Verfasser:}

Dozent Dr. med. habil. Gudrun Wiedemann Klinische Chemie und Laboratoriumsdiagnostik des Klinikums Erfurt Nordhäuser Straße 74 99089 Erfurt 